\title{
Téoros
}

Revue de recherche en tourisme

\section{La ville à la campagne?}

\section{Marcel Samson}

Volume 10, numéro 1, mars 1991

\section{Tourisme et environnement}

URI : https://id.erudit.org/iderudit/1079816ar

DOI : https://doi.org/10.7202/1079816ar

Aller au sommaire du numéro

\section{Éditeur(s)}

Université du Québec à Montréal

\section{ISSN}

0712-8657 (imprimé)

1923-2705 (numérique)

Découvrir la revue

Citer cet article

Samson, M. (1991). La ville à la campagne ? Téoros, 10(1), 26-28.

https://doi.org/10.7202/1079816ar d'utilisation que vous pouvez consulter en ligne.

https://apropos.erudit.org/fr/usagers/politique-dutilisation/ 
Dans un article publiê au début des années $1980^{(1)}$, nous affirmions que ${ }^{54}$ comme la plupart des pays industrialisés, le Canada (et le Québec) a connu ces dernières années un renversement des tendances démographiques séculaires. En effet, les courants migratoires qui ont transformé un Québec rural en un Québec urbain ne se font plus sentir depuis le début des années $1970^{\circ}$. Nous nous étions alors appuyé sur une étude des démographes Mongeau et Termote ${ }^{2)}$ qui révélait que le recensement de 1976 montrait que pour la première fois dans l'histoire canadienne, les régions rurales ont connu un taux d'accroissement plus élevé que les régions urbaines et que la difference entre les deux taux était encore plus grande entre 1976 et 1981 . Le terme contre-urbanisation a êté employé justement parce que la croissance de population était plus rapide en zone rurale qu'en zone urbaine ${ }^{\text {(3) }}$ et que cette notion permettait de distinguer de la rurbanisation ou de la simple extension spatiale, de la suburbanisation ou de la métropolisation. Or, Termote et Mongeau, dans $1^{\prime}$ étude déjà mentionnée, concluaient en affirmant que "la contreurbanisation existe au Québec, mais elle se localise essentiellement, quoique de moins en moins, dans les régions adjacentes aux grands centres urbains. Elle touche en fait très peu les régions éloignées des grands centres, et elle se manifeste surtout dans les comtés déjà fortement urbanisés ${ }^{n+(4)}$. On pouvait percevoir que les auteurs laissaient transparaître un certain scepticismequantà la réalité de la contre-urbanisation. Aujourd'hui, il ne faitpresque plus dedoute que la contre-urbanisation entendue dans son sens le plus étroit n'a vraiment pas existé au Québec ni même au Canada. Des chercheurs de l' Université Guelph mentionnent mềme que là où les taux de croissance ruraleont, selon toute vraisemblance, dépassé les taux urbains dans les années 1970 , "l'ampleur de ce virage resulte sans doute d'exagérations dans les données du recensement attribuables aux effets de délimitation et de reclassification" ${ }^{\text {nis? }}$. Plus encore, tout porte à croire que finalement on est en présence d'une simple décongestion des

\footnotetext{
Marcel Samson est professeur au Departement d'etudes urbaines et touristiques de l'Universite du Québec à Montréal
}

centres urbains. Autrement dit, la contreurbanisation n'aurait jamais existé comme telle au Québec. Au contraire, on parle maintenant du déclin du monde rural, de la désertification des régions rurales... Plus de 700 villages seraient menacés de mort. Alors, si la contre-urbanisation a déjà existế, c'est bel et bien terminé!

Par ailleurs, nous avons toujours soutenu que les régions contiguës aux centres urbains et particuliêrement Montréal et Québec, où la villégiature en résidences secondairesest importante, constituent des zones privilégiées d'implantation d'ex-urbains. Une étude exploratoire effectuće en 1980 sur quelques municipalités des Laurentides montréalaises nous avaitconvaincus que ce mouvement était bien réel et qu'il se poursuivrait probablement dans les années 1980 et 1990. En affirmant cela, nous ne faisions que confirmer les premières observations faites au Québec sur l'exode urbain au cours des années 1970. Rappelons qu'à partir de ses travaux sur le sujet, le géographe Brunet soulignait qu ${ }^{3 *}$ en moyenne, $17,5 \%$ de la population des municipalités rurales non-métropolitaines habitaiten ville en $1971^{1 "(6)}$. Il ajoutait alors qu'en direction nord de l'agglomération de Montréal, "l'influence métropolitaine se fait sentir plus loin et avec plus d'intensité qu'au sud, en particulier par l'intermédiaire des migrations hebdomadaires et saisonnières de villégiature des Montréalais vers une mul= titude de résidences secondaires ${ }^{21}(T)$. Rappelons aussique des chercheurs de l'Office de planification et de développement du Québec avaient à la même période émis l'hypothese que la localisation des zones d'accroissement démographique au nord de Sainte-Scholastique et Saint-Jérổme "s'expliquerait pour une grande part grâce au phénomène de villégiature, c'est-à-dire fixation d'une population nouvelle, principalement citadine, par la transformation de résidences secondaires en résidences permanentes et par l'offre de services touristiques $^{n(\text { (w) }}$. En fait, ces observations viennent confirmer les nouvelles conduites qui se sont développées en rapport avec la résidence secondaire à partir du début des années 1960. Quelques facteurs liés à l'évolution socio-économique de la société québécoise ont favorisé ces changements.

\section{De nouvelles conduites}

Ainsi, de 1957 à 1961, leQuébec a vécu une période destagnation économique qui s'est traduit par un taux de chômage qui a atteint $9,3 \%$ de la population active en 1961. Mais cette crise conjoncturelle aété suivie d'une forte expansion économiquequi a connu un sommet entre 1962 et 1967. Au même moment, le parc de résidences secondaires augmentait de façon considérable. Par ailleurs, si la croissance démographique marque le pas, il y a formation de plusieurs nouveaux ménages, résul tat du baby boom, et donc une demande résidentielle très forte qui se traduira par l'expansion de la banlieue. Plus encore, l'augmentation du parc automobile, la construction d'un nouveau réseau routier, particulièrement le prolongement de l'autoroute du Nord, déjà inauguré en 1955, et. l'augmentation généralisée de la consommation des ménages auront un impact immediat sur l'essor de la villégiature. Des résidences secondaires sont maintenant converties en résidences principales par des retraités ou par des ménages qui ont une occupation, des revenus et une liberté de déplacement suffisante pour faire de certains espaces des nouvelles banlieues plus ou moins éloignées de la métropoleou des centres urbains régionaux les plus importants. De plus, $1^{\prime}$ étalement de la fréquentation sur une base annuelle tend à se répandre. Mais cela ne se fait pas uniformément, les facteurs influençant ces conduites nouvelles relèvent du potentiel naturel de ces espaces, il va de soi, mais aussi et surtout des facilites de communication et donc de la distance-temps des centres urbains de services et d'emplois. Ce même comportement a été observé ailleurs en Amérique du Nord et dans les sociétés industrialisées en général. Hugh D. Clout, dans un article important sur le sujet au début des années 1970, faisait les remarques suivantes:

Second homes merit geographical attention for two main reasons. First, they add new and distinctive features to the already complex assemblage of residential areas around urban cores, thereby transporting "the city" and its inhabitants into the countryside for substantial periods each year. Second, the seasonal presence of secondhome occupants gives rise to important 


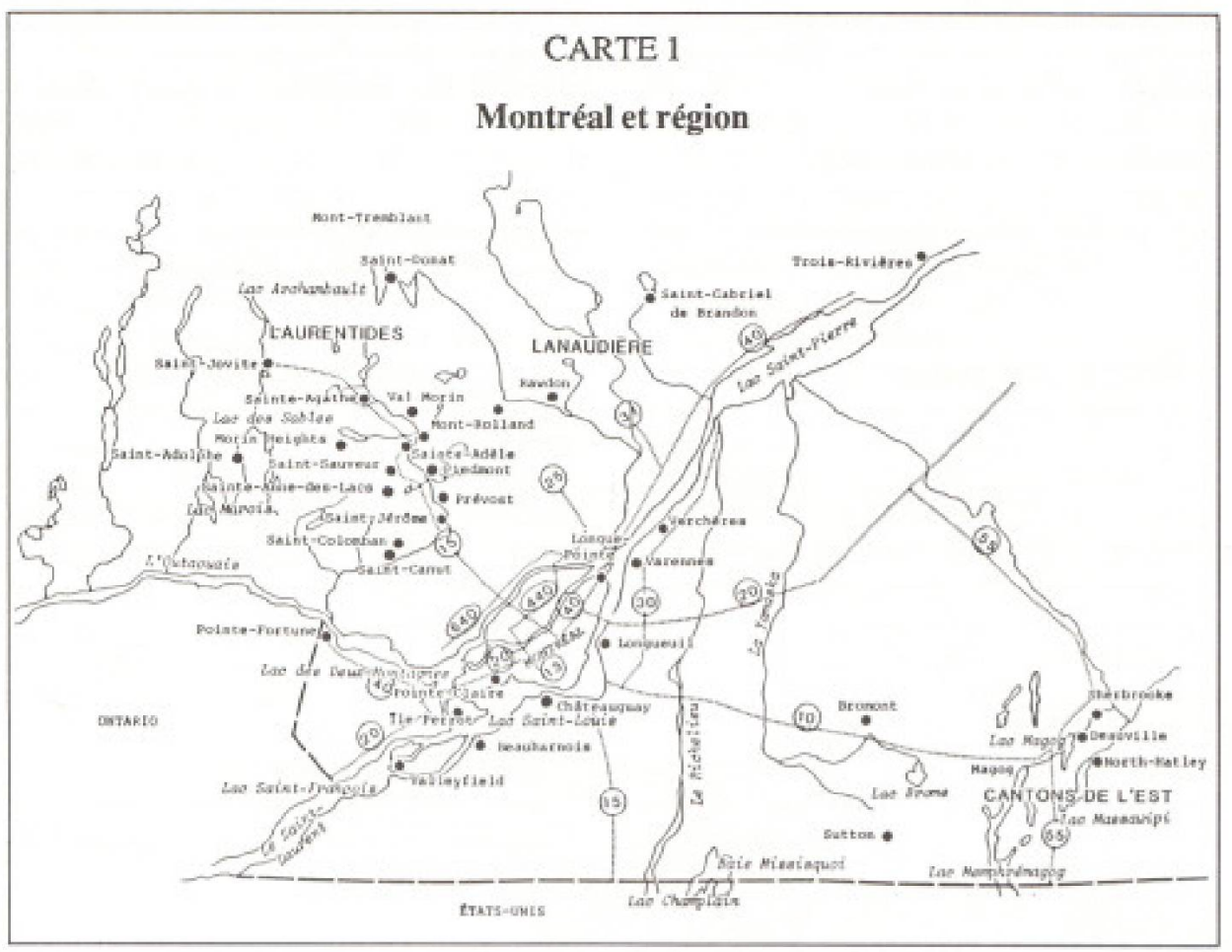

social and economic changes in host communities ${ }^{(9)}$.

Ainsi,ces périodes de plusen plus fréquentes d'utilisation de la résidence secondaire ont entraîné pour plusieurs propriétaires la mutation de la résidence secondaire à la résidence principale de sorte que la banlieurisation n'est plus simplement "saisonnière", terme employé par Clout, mais elle est bel et bien devenue une extension de la ville centrale. Pour illustrer notre propos, nous retiendrons, à l'intérieur du corridor le plus fréquenté des Laurentides montréalaises, les agglomérations de la vallé de Saint-Sauveuret de la municipalité de Sainte-Adèle.

\section{La campagne urbanisée: Saint-Sauveur et Sainte-Adèle}

La vallée de Saint-Sauveur est $1^{\text {texemple }}$ par excellence de l'appropriation montréalaise sur l'espace ruralenvironnant. Il faut savoir d'abord que la vallée de SaintSauveur comprend trois entités administratives et spatiales distinctes. Ce découpage remonte aux années 1920 alors qu"un différend concernant la construction d'un aqueduc (manifestation de l'infrastructure urbaine) provoquait l'éclatement du territoire et aboutissait à l'existence de Saint-Sauveur-des-Monts comme municipalité de village, à SaintSauveur paroisse et à Piedmont comme municipalité sans désignation. Cette rupture survenue dès le premier quart de siecle ne sera pas sans conséquences sur le développement et l'aménagement de l'espace quelque 60 ans plus tard.

Au XIXe siècle, comme à bien des endroits au Québec, le village s'est développé principalement autour de l'Église de la municipalité de Saint-Sauveur mais s'est. aussi étendu sur les rives de la Rivière du Nord où se situe Piedmont. C'est donc sur cet axe linéaire touchantà Saint-Sauveuret à Piedmont que s'est localisée d'abord la vie de la vallée. Mais, des la fin du XIXe siècle, un deuxième axe, la rue de la Gare, centréesur le prolongementde la voie ferrée et liće à l'étalement de la villégiature et aux activités de sport d'hiver s'est développé. Ainsi, à cette même période, hôtels et villas sont présentes sur la rue principale et le ski de descente commence son essor. Dans un deuxième temps, les bordures des plans d'eau se privatisent, l'un après l'autre si bien qu'aujourd'hui tous les lacs sont occupés par un chapelet de résidences secondaires ou permanentes. Enfin, plus récemment, de véritables villages résidentiels ainsi que des ensembles de copropriétés de villégiature se sont érigés dans le fond de la vallée ou au pied des pistes de ski sous l'action des promoteurs "industriels" venus de la ville, selon les termes de Lundgren ${ }^{[10)}$. Ainsi, au développement linéaire de l'espace villageois a succédé un développement tout azimut. Le noyau villageois ancien est passé de la fonction habitation à la fonction commerciale; les maisons de la rue principale et de la rue de la Gare ont été progressivement transformées en commerces. De nouveaux quartiers résidentiels calqués sur la forme proprement urbaine se sont greffes à ce noyau ancien. Et, pour boucler la boucle de cette rurbanisation qui atteint son apogée à partir du milieu des années 1970, on voit surgir comme dans la banlieue classique une série de centres d'achats ou dans des termes plus élégants, des "galeries commerciales": La Galerie des Monts construite en 1977, le Faubourg Saint-Sauveur en 1986, les Promenades Saint-Sauveur en 1987 et les Trois Villages (raison commerciale très évocatrice!) en 1988.

De 1961 à 1981, la population de la vallée a connu une augmentation générale de $65,9 \%$ passant de 2352 à 6405 alors que l'augmentation moyenne dans les municipalités au Québec s'établit pour la même période à $24,7 \%$. Si l'augmentation la plus forte s'est faite sur le village entre 1976 et 1981, c'est sur Saint-Sauveur paroisse entre 1981 et 1986 et sur Piedmont depuis 1986 que la hausse a été la plus sensible. Dans ces deux derniers cas, $c^{\dagger}$ est. l'action des promoteurs industriels et l'érection de nombreuses copropriétés de villégiature (condominiums) qui a marqué ces périodes. Mais à ce niveau microspatial, il est difficile d'évaluercorrectement l'ampleur du mouvement de rurbanisation. Il faut penser plutôt que c'est l'ensemble des déplacementsdes personnes, aussi bien excursionnistesque population de résidents secondaires, semi-permanents ou permanents, de même que le volume d'activités commerciales et de services qui reflètent particulièrement la situation de cette zone par rapport à la région métropolitaine.

Le morcellement du territoire qui aboutit du même coup à un morcellement des décisions et des responsabilités a eu un impact desastreux sur l'environnement. Qu'il suffise de dire que jusqu'en 1981, à l'exception de la municipalité de SaintSauveur concernant la sauvegarde du patrimoine architectural, le contrôle de l'utilisation du sol était pratiquement inexistant ou, à tout le moins, la réglementation était très permissive. Et jusqu'à récemment, les divergences de législation entre les trois entités territoriales portaient sur plusieurs aspects tels que l'affichage, la politique concernant l'adduction d'eau et l'évacuation des eaux usées, le contrôle de la construction résidentielle et l'attitude générale face à l'environnement. Comme dans les autres régionsdu Québec, la municipalité régionale de comté des Pays d'en Haut a depuis 1986 un schéma d'aménagement; au mềme moment, l'Association touristique des Laurentides déposait son plan de développement touristique. Ces deux instruments de planification ne peuvent être efficaces que s'ils sont le produit d'une 
concertation véritable entre les différents pouvoirs décisionnels et, particulièrement, entre le secteur privế et le secteur public. Or, force est d'admettre que les efforts des uns sont anéantis par les politiques divergentes des autres; la rivalité prend le pas sur la concertation dès que des intérêts économiques ou politiques sont en cause. Les véritables enjeux sont finalement obscurcis par des visions de développement à très court terme.

\section{Sainte-Adèle: un pôle de développement}

Au coeur des Laurentides montréalaises, c'est à partir de 1945 que l'activité touristique prend un tournant définitif dans l'axe Saint-Sauveur et Sainte-Agathe, plus au nord, avec Sainte-Adèle comme pôle de développement. Les années 1960, avec l'ouverture du nouveau tronçon de l'autoroute des Laurentides qui relie dorénavant Sainte-Adèle, confirment définitivement la vocation touristique centrale du corridor Saint-Sauveur/SainteAdèle qui devient une zone d'accueil pour une migration de population quotidienne, saisonnière ou de fins de semaine de plus en plus importante. Par la suite, le premier choc pétrolier du début des années 1970 ne ralentira pas la croissance de l'activité touristique qui ne sera vraiment freiné, et assez durement, durant la récession qui marque les années 1981-1982. La reprise économique et surtout l'injection massive par le secteur public de capitaux pour la modernisation des centres de ski et, plus généralement, dess infrastructures reliées à l'industrie touristique débouchera sur la mise en chantier de nombreux projets résidentiels. Côté population, les tendances observées reflètent bien ce qui s'est produit ailleurs dans la zone. Ainsi, de 1971 à 1976 , la population s'est accrue de $9,6 \%$, passant de 3818 à 4186 résidents permanents, et de $11,7 \%$ entre 1976 à 1981 alors que la population atteindra 4675 . Après un freinage et même une diminution entre 1981 et 1986, la population depuis lors s'élèverait à 5000 résidents selon une estimation de la municipalité alors que la population de résidents secondaires à I'intérieur des limites territoriales municipale pourrait bien atteindre le double en termes, cette fois-ci,decapacité d'accueil de l'habitat de villégiature ${ }^{(11)}$.

Sous l'aspect économique, les secteurs primaire et secondaire sont à toutes fins utiles absents de Sainte-Adèle au profit du secteur tertiaire, alimenté par le système récréo-touristique. À titre indicatif, mentionnons que selon l'Office de planification et de développement du Québec, le secteur tertiaire a accaparé la plus grande part des investissements dans la région des Laurentides avec des montants de 81,5 millions de dollars en 1985,74 millions de dollarsen $1986 \mathrm{et} 190,8$ millions de dollars en 1987, et ceschiffres n' incluent pas un ensemble de projets de moindre envergure ${ }^{(12)}$. De ces investissements, $c^{2} e s t$ la MRC des Pays d'en Haut qui tient le haut du pavé avec des sommes importantes investies dans les secteurs hébergement et restauration, divertissementet loisirs. Cette activité économique a eu un effet direct sur le parc de résidences secondaires qui atteignait environ 1669 unités en 1989 alors qu'il se situait autour de 845 en 1981 . Entre 1986 et 1989 seulement, la hausse qui s'établit à $55,4 \%$ est très révélatrice de l'action despromoteurs qui ont littéralement inondé le marché immobilier de projets de type "condo". Cette action a eu aussi pour effet de remplir l'espace résiduel entre le noyau villageois ancien et les résidences de villégiature privée qui, là comme ailleurs, avaient déjà envahi les rives des plans d'eau avoisinants. De sorte que cetélargissement du périmètre urbain a également déplacé l'activité commerce en favorisant l'implantation d'un centre commercial regroupantquelques vingt-cinq commerces spécialisés, hors du périmètre urbain traditionnel. Ainsi, l'effet banlieue s'est-il fait sentir de façon aussi nette que dans la vallée de Saint-Sauveur. En fait, l'abondance des commerceset des services du secteur privé comme du secteur public ont fait de Sainte-Adèle un pôle régional à I'intérieur de la MRC des Pays d'en Haut. A un point tel que, sous certains aspects, on peut affirmer que cette municipalité est mieux équipée et mieux desservie qu"un centre urbain de plus grande taille comme Saint-Jérôme.

\section{Quel avenir?}

Nous savons que l'implantation de la villégiature privée autour des plans d'eau s*est fait pratiquementsans réglementations et parfois dans l'anarchie la plus totale. À cet état de fait et à la lumière de l'analyse que l'on vient d'effectuer, certaines zones etc'estlecas del'axeSaint-Sauveur/SainteAdèle ont connu une urbanisation rapide ces dernières années. Les outils législatifs dont on s'est dotés récemment tels la Loi sur l'aménagement et l'urbanisme et la Loi sur la qualité de l'environnement n'ont pu freiner l'appétitde certains promoteurs qui, contraints parfois par la Loi sur la protection des terres agricoles, se sont rabattus sur des espaces qui permettaient de transposer le schéma urbain sans contraintes. Ces tendances à l'urbanisation qui se sont manifestées, nous l'avons déjà mentionné, dès les années 1960 et qui ont connu une accélération marquée dans les années 1980 devraient connaître une période d'essoufflement dans les années 1990. La disponibilité des espaces se faisant de plus. en plus rare a eu pour conséquence d'augmenter la valeur du parc immobilier résidentieletd'en restreindre l'accessibilité aux plus nantis, c'est-ầ-dire à ceux-là même qui pourrontencore s'offrir dans les années 1990 une résidence secondaire ou qui choisiront d'y habiter en permanence parce que leur situation occupationnelle leur permettra. Pour d'autres, ces choix seront certainement plus difficiles; le krach de 1988, la récession du début de la présente décennie, le poids plus lourd de taxation imposé au palier municipal conduiront à des perspectives difficiles pour ces municipalités dont le secteur récréotouristique est le moteur principal. En terme environnemental, rien ne sert de $s^{3}$ appesantir sur les erreurs passées, il faut plutôt fonder l'avenir de ces régions sur une vision la plus claire possible des quelques enjeux locaux et régionaux qui marqueront la fin de ce siècle.

\section{Notes explicatives}

(1) SAMSON, Marcel, "contre-urbanisation et résidences secondaires". Téoros, vol, 2, no 3 , octobre 1983, pp. 13-16.

(2) TEAMOTE, Marc G. et Jael MONGEAU, "L'ampleur de la contre-urbanisation au Québec ", textemodifié d'une communication présentée au Colloque sur Íexode urbain, Departement de geographie. Université de Montreal, avril 1962

(3) BERRY, Brian $J$ - L. Ied I. "Urbanisation and CounterUrbanisation", vol. 11. Urban Affairs Annual Review, Beverly Hills, California, Sage Publications, 1976.

(4) TERMOTE, M.G. et Jaël MONGEAU, op. cit, p. 22.

(5) JOSEPH Alun E KEDDIE, Fhilip D et Barry SMIT, "Unravelling the Population Tumaround in Rural Canada" "The Canadian Geographer/Le gotographe canadien, wol. 32 , no $1,1988, p p .17$ 30

(6) BRUNET. Yves, "L'exode urbain dans la région de Montreal". Notes et documents 80-05, Departement de geographie. Université de Montreal, 1990, p. 11

(7) BRUNET, Yves, op. cit. p. 9

(B) FAUTEUX, Martial et al. "Notes concernant la croissance récente de l'arrière-pays et la diffusion delurbanisation dan la région montréalaise" "OPDQ. Montreal, 1977 , p. 34

(9) CLOUT, Hugh D., "the Growth of second-home Ownership: An Example of seasonal suburbanisation". J.H. JOHNSON ed, suburban Growth geographical Processes at the Edge of the Wostern city. London, John Wiloy, pp. 101125

(10) Voir Jean 0. Lundgren, "Tourism Destination Development and Problems of Management; Case Lake Memohremagog Region". Teoros, vol. 7, no 2. 1968 , pp. 11-16.

(11) BERTRAND, Jean-François, Indice du développement touristique et da la villégiature sur la situation urbaine et financiere de SainteAddelo, mémoire de maítrise en analyse et gestion urbaines, UOAM, 1990

(12) OFFICE DE PLANIFICATION ET DE DÉVELOPPEMENT DU Quebec, Bilan socioéconomique de la région des Laurentides, Ouebec, Gouvernement du Québec, 1987. 